International Journal of Science and Research (IJSR)

ISSN (Online): 2319-7064

Index Copernicus Value (2015): 78.96 | Impact Factor (2015): 6.391

\title{
Case Report: A 69 - Year - Old Women with Stomach Cancer
}

\author{
Supriya $^{1}$, Rupali Kumari ${ }^{2}$ \\ ${ }^{1}$ Ex. Registered Dietician Intern, Apollo Hospital, Bangalore, Karanataka, India \\ ${ }^{2}$ Dietician, Jagdish Memorial Hospital, Patna, Bihar, India
}

\section{Background}

Stomach cancer, also called gastric cancer, a disease characterized by abnormal growth of cells in the stomach. Ninety-five percent of malignant stomach cancers develop from epithelial cells lining the stomach. These tumours are called adenocarcinomas. Gastric cancer is a disease in which cancer cells form in the lining of the stomach.

\section{Case Presentation}

Patient was 69 yr old lady admitted in June 2013 with diagnosis of carcinoma of stomach. Histopathology report showed - Adenocarcinoma with well differentiated type. There was past medical history of loss of appetite due to which there was loss of weight -3 to $4 \mathrm{~kg}$ since three months but there is no history of abdomen pain, loose stools, vomiting, fever, cold, throat pain and there was no family history of stomach cancer. She never smoked and didn't drink alcohol.

\section{Investigation}

On examination, patient blood pressure was $120 / 80$, pulse was 82 beats per minute and her respiratory rate was 20 times per minute, temperature was a febrile and the body mass index was $15 \mathrm{~kg} / \mathrm{m}^{2}$. Result of complete blood count was not normal.

\section{Biochemical Parameters}

\begin{tabular}{|c|c|c|}
\hline Parameter & Result & Normal Range \\
\hline Haemoglobin & $10.3 \mathrm{gm} / \mathrm{dl} *$ & $11.5-14.5$ \\
\hline $\mathrm{WBC}$ & 5.9 thousands/cumm & $4-11$ \\
\hline $\mathrm{RBC}$ & 4.0 millions $/ \mathrm{cmm}^{*}$ & $4.5-5.5$ \\
\hline Platelet count & 242 thousands/cumm & $150-450$ \\
\hline Polymorph & $54 \%$ & $40-75$ \\
\hline Lymphocyte & $33 \%$ & $20-45$ \\
\hline Monocyte & $7 \%$ & $2-10$ \\
\hline Eosinophil & $6 \%$ & $1-6$ \\
\hline Basophil & $0 \%$ & $0-1$ \\
\hline Packed cell volume & $34 \%$ & $36-46$ \\
\hline Serum alkaline phosphatase & $71 \mathrm{U} / \mathrm{L}$ & $30-120$ \\
\hline Serum bilirubin total & $0.38 \mathrm{mg} / \mathrm{dl}$ & $0.2-1.2$ \\
\hline S. bilirubin direct & $0.06 \mathrm{mg} / \mathrm{dl}$ & $0.0-0.4$ \\
\hline S. bilirubin indirect & $0.32 \mathrm{mg} / \mathrm{dl}$ & $0.1-1.0$ \\
\hline S. Total protein & $4.8 \mathrm{~g} / \mathrm{dl} *$ & $6.0-8.5$ \\
\hline S. Albumin & $2.3 \mathrm{~g} / \mathrm{dl} *$ & $3.5-52$ \\
\hline S. Globulin & $2.5 \mathrm{~g} / \mathrm{dl}$ & $2.0-4.0$ \\
\hline A/G Ratio & 0.9 & $0.8-2.0$ \\
\hline S. SGPT & $25 \mathrm{U} / \mathrm{L}$ & $5-40$ \\
\hline S. SGOT & $8 \mathrm{U} / \mathrm{L}$ & $5-45$ \\
\hline S. Gamma GT & $7 * \mathrm{U} / \mathrm{L}$ & $10-45$ \\
\hline S. Urea & $19 \mathrm{mg} / \mathrm{dl}$ & $15-45$ \\
\hline S. Creatinine & $0.81 \mathrm{mg} / \mathrm{dl}$ & $0.81-1.44$ \\
\hline S. Sodium & $139 \mathrm{mmol} / \mathrm{L}$ & $135-145$ \\
\hline S. Potassium & $4.6 \mathrm{mmol} / \mathrm{L}$ & $3.5-5.5$ \\
\hline S. Bicarbonate & 25 & $22-29$ \\
\hline Plasma glucose & $116 \mathrm{mg} / \mathrm{dl}$ & $70-140$ \\
\hline $\mathrm{T} 3$ & $3.87 \mathrm{pg} / \mathrm{ml}$ & $2.5-3.9$ \\
\hline $\mathrm{T} 4$ & $0.79 \mathrm{ng} / \mathrm{dl}$ & $0.61-1.12$ \\
\hline TS4 & $2.56 \mathrm{IU} / \mathrm{ml}$ & $0.34-5.6$ \\
\hline
\end{tabular}




\section{International Journal of Science and Research (IJSR) \\ ISSN (Online): 2319-7064}

Index Copernicus Value (2015): 78.96 | Impact Factor (2015): 6.391

\section{Management}

After thorough pre-operation evaluation, next day patient underwent total gastrectomy with Roux-en-Y esophagojejunostomy. Patient was shifted to SICU after surgery. Patient developed sepsis with thrombocytopenia and coagulopathy for which she was treated. The antibiotics were escalated according to the blood culture and sensitivity reports.

\section{Medication History}

\begin{tabular}{|c|c|c|c|}
\hline Medicine & Dose & Frequency & Purpose \\
\hline Inj.Emeset & $4 \mathrm{mg}$ & $\mathrm{SOS}$ & $\begin{array}{c}\text { Prevent nausea and } \\
\text { vomiting }\end{array}$ \\
\hline Inj.Pantodac & $40 \mathrm{mg}$ & $1-1-1-1$ & Antacid \\
\hline Inj.Supracef & $1 \mathrm{gm}$ & $1-0-1$ & Antibiotic \\
\hline Inj.Metrogyl & $\begin{array}{c}500 \\
\mathrm{mg}\end{array}$ & $1-0-1$ & Antibiotic \\
\hline $\begin{array}{c}\text { Budecort } \\
\text { Nebulisation }\end{array}$ & $\begin{array}{c}0.15 \\
\mathrm{mg}\end{array}$ & $1-0-1$ & Bronchodilator \\
\hline $\begin{array}{c}\text { Duolin } \\
\text { Nebulisation }\end{array}$ & $2.5 \mathrm{ml}$ & $\mathrm{Q} 8 \mathrm{hrly}$ & Bronchodilator \\
\hline $\begin{array}{c}\text { Inj. Myo } \\
\text { Pyrocate }\end{array}$ & $1 \mathrm{amp}$ & Stat & Muscle Relaxant \\
\hline Inj.Perfalgam & $1 \mathrm{gm}$ & $1-0-1$ & Pain killer \\
\hline Inj.Voveran & $75 \mathrm{mg}$ & Stat & Painkiller \\
\hline Tab.Pantop & $40 \mathrm{mg}$ & $1-0-0$ & Anta acid \\
\hline Cap.Providac & & $1-1-1$ & Anti Diarrhoeal \\
\hline Tab.Ceftum & $\begin{array}{c}500 \\
\mathrm{mg}\end{array}$ & $1-0-1$ & Antibiotic \\
\hline
\end{tabular}

\section{Conclusion}

Patient developed pneumonia and was intubated in view of respiratory failure, also had acute renal failure for which patient was on lasix. Eventually underwent tracheostomy after two weeks and was on ventilator. Patient was initial put on TPN then was started on NJ feeds and oral feeds. Patient had loose stools issues, sepsis with septic shock, acute respiratory distress syndrome, hypoalbuminemia and chest infection. After four weeks, patient developed bradycardia and hypotension and gradually went to cardiac arrest. Inspite of all resuscitative measures patient could not be reviewed and declared dead. 